\title{
SPLINE COALESCENCE HIDDEN VARIABLE FRACTAL INTERPOLATION FUNCTIONS
}

\author{
A. K. B. CHAND AND G. P. KAPOOR \\ Received 13 February 2006; Revised 13 July 2006; Accepted 8 August 2006
}

This paper generalizes the classical spline using a new construction of spline coalescence hidden variable fractal interpolation function (CHFIF). The derivative of a spline CHFIF is a typical fractal function that is self-affine or non-self-affine depending on the parameters of a nondiagonal iterated function system. Our construction generalizes the construction of Barnsley and Harrington (1989), when the construction is not restricted to a particular type of boundary conditions. Spline CHFIFs are likely to be potentially useful in approximation theory due to effects of the hidden variables and these effects are demonstrated through suitable examples in the present work.

Copyright (C) 2006 A. K. B. Chand and G. P. Kapoor. This is an open access article distributed under the Creative Commons Attribution License, which permits unrestricted use, distribution, and reproduction in any medium, provided the original work is properly cited.

\section{Introduction}

The class of self-affine attractors was introduced by Mandelbrot [12], and its construction through iterated function systems (IFSs) was proposed by Hutchinson [11]. By using IFSs, Barnsley [1,2] and Barnsley et al. [3] introduced the concept of fractal interpolation functions (FIFs), that are used for approximating naturally occurring functions, for example, speech signals [13], seismic data [10], electrocardiograms [15], images [5], which show some kind of geometrical self-similarity under magnification. For instance, in a case of a speech signal, the approximation is achieved by replacing the original data with the set of generating parameters (which are actually matrix elements of certain contractive affine mappings) used for generating a suitable FIF. The non-self-affine functions are approximated by using hidden variable FIFs studied by Barnsley [2], Barnsley et al. [3], and Massopust [14]. In [7], the projection of the graphs of vector-valued functions or attractors from a nondiagonal IFS is used to approximate self-affine or

Hindawi Publishing Corporation Journal of Applied Mathematics Volume 2006, Article ID 36829, Pages 1-17 DOI 10.1155/JAM/2006/36829 
non-self-affine functions simultaneously by introducing the concept of constrained free variables. The smoothness analysis of CHFIF [6] gives that the deterministic construction of functions having modulus of continuity $O\left(|t|^{\mu}(\log |t|)^{m}\right)(m$ is an integer, $m \geq$ 0 , and $0<\mu \leq 1$ ) is possible.

The construction of self-affine spline FIF with only initial endpoint boundary conditions is given by Barnsley and Harrington [4]. However, generating spline FIFs with general boundary condition is not possible in their construction due to a particular recurrence relation between the matrices. Some results on the convergence of cubic spline FIFs on uniform meshes are found in [17]. The general construction of spline FIFs and their convergence results are developed recently in $[6,8,9,16-19]$. A CHFIF can be integrated successively in order to get smoother interpolation functions that generalize classical splines. The resulting smooth functions are also attractors of appropriate nondiagonal IFSs but they interpolate different data in general. The inverse problem is the construction of a spline CHFIF that interpolates the given data. Although spline CHFIFs are not actually fractals, certain derivatives of these functions are typical fractal functions that may be self-affine or non-self-affine in nature. The fractal dimension of a suitable derivative of spline CHFIFs can be aptly used as a quantifying parameter for various complex phenomena.

In the present paper, the existence of interpolating $C^{r}$-CHFIF for given real data with all possible boundary conditions is established by studying the calculus of vector-valued $C^{1}$-FIF. The functional relations of $C^{r}$-CHFIF at the endpoints of the interval with joinup conditions and interpolation conditions give a system of equations whose solution determines the polynomial coefficients in the construction of a nondiagonal IFS. The advantage of such a construction is that with suitable choices of the hidden variables for prescribed data and given boundary conditions, one can construct self-affine or non-selfaffine spline function according to the need of an experiment from a number of available spline CHFIF.

The organization of this paper is as follows: in Section 2, preliminaries concerning CHFIF are discussed. The basic calculus of vector-valued $C^{1}$-FIF is studied in Section 3. The spline CHFIF with general boundary conditions is constructed in Section 4 . The effects of hidden variables on spline CHFIFs are demonstrated in Section 5 through various examples.

\section{Preliminaries of CHFIF}

Suppose that the real data to be interpolated is given by $\left\{\left(x_{n}, y_{n}\right) \in \mathbb{R}^{2}: n=0,1,2, \ldots, N\right\}$, where $-\infty<x_{0}<x_{1}<\cdots<x_{N}<\infty$. In order to construct an interpolation function $f_{1}$ : $\left[x_{0}, x_{N}\right] \rightarrow \mathbb{R}$ such that $f_{1}\left(x_{n}\right)=y_{n}$, for all $n=0,1,2, \ldots, N$, consider a generalized set of data $\left\{\left(x_{n}, y_{n}, z_{n}\right) \in \mathbb{R}^{3} \mid n=0,1,2, \ldots, N\right\}$, where the $z_{n}, n=0,1,2, \ldots, N$, are finite real numbers. The following notation is needed in the construction of CHFIF: $I=\left[x_{0}, x_{N}\right]$, $I_{n}=\left[x_{n-1}, x_{n}\right], g_{1}=\operatorname{Min}_{n} y_{n}, g_{2}=\operatorname{Max}_{n} y_{n}, h_{1}=\operatorname{Min}_{n} z_{n}, h_{2}=\operatorname{Max}_{n} z_{n}$, and $K=I \times D$, where $D=J_{1} \times J_{2}, J_{1}, J_{2}$ are suitable compact sets in $\mathbb{R}$ such that $\left[g_{1}, g_{2}\right] \times\left[h_{1}, h_{2}\right] \subset D$. Let $L_{n}: I \rightarrow I_{n}$ be a contractive homeomorphism and let $F_{n}: K \rightarrow D$ be a continuous 
vector-valued function such that

$$
\begin{gathered}
L_{n}\left(x_{0}\right)=x_{n-1}, \quad L_{n}\left(x_{N}\right)=x_{n}, \\
F_{n}\left(x_{0}, y_{0}, z_{0}\right)=\left(y_{n-1}, z_{n-1}\right), \quad F_{n}\left(x_{N}, y_{N}, z_{N}\right)=\left(y_{n}, z_{n}\right), \\
d\left(F_{n}(x, y, z), F_{n}\left(x^{*}, y, z\right)\right) \leq c\left|x-x^{*}\right| \\
d\left(F_{n}(x, y, z), F_{n}\left(x, y^{*}, z^{*}\right)\right) \leq s d_{E}\left((y, z),\left(y^{*}, z^{*}\right)\right),
\end{gathered}
$$

for all $n=1,2, \ldots, N$, where $c$ and $s$ are positive constants with $0 \leq s<1,(x, y, z),\left(x^{*}, y, z\right)$, $\left(x, y^{*}, z^{*}\right) \in K, d$ is the sup norm on $K$, and $d_{E}$ is the Euclidean metric on $\mathbb{R}^{2}$. For defining the required CHFIF, the functions $L_{n}$ and $F_{n}$ are chosen to be of the form $L_{n}(x)=a_{n} x+b_{n}$ and

$$
F_{n}(x, y, z)=A_{n}(y, z)^{T}+\left(p_{n}(x), q_{n}(x)\right)^{T}=\left(F_{n}^{1}(x, y, z), F_{n}^{2}(x, z)\right)^{T},
$$

where $A_{n}$ is an upper triangular matrix $\left(\begin{array}{cc}\alpha_{n} & \beta_{n} \\ 0 & \gamma_{n}\end{array}\right)$ and $p_{n}(x), q_{n}(x)$ are continuous functions having at least two unknowns. We choose $\alpha_{n}$ as free variable with $\left|\alpha_{n}\right|<1$ and $\beta_{n}$ as constrained free variable with respect to $\gamma_{n}$ such that $\left|\beta_{n}\right|+\left|\gamma_{n}\right|<1$. The generalized IFS that is needed for construction of CHFIF corresponding to the data $\left\{\left(x_{n}, y_{n}, z_{n}\right) \mid n=\right.$ $0,1, \ldots, N\}$ is now defined as

$$
\left\{\mathbb{R}^{3} ; \omega_{n}(x, y, z)=\left(L_{n}(x), F_{n}(x, y, z)\right), n=1,2, \ldots, N\right\} .
$$

It is known [3] that the IFS defined in (2.4) associated with the data $\left\{\left(x_{n}, y_{n}, z_{n}\right), n=\right.$ $0,1, \ldots, N\}$ is hyperbolic with respect to a metric $\rho$ on $\mathbb{R}^{3}$ equivalent to the Euclidean metric. In particular, there exists a unique nonempty compact set $G \subseteq \mathbb{R}^{3}$ such that

$$
G=\bigcup_{i=1}^{N} \omega_{i}(G) .
$$

The existence of a unique vector-valued function $f$ that interpolates the generalized interpolation data by establishing the graph of $f$ that equals the attractor $G$ of the generalized IFS is given in the following proposition.

Proposition 2.1 [3]. The above attractor $G$ of the IFS defined in (2.4) is the graph of the continuous vector-valued function $f: I \rightarrow D$ such that $f\left(x_{n}\right)=\left(y_{n}, z_{n}\right)$ for all $n=1,2, \ldots, N$, that is, $G=\{(x, y, z): x \in I$ and $f(x)=(y(x), z(x))\}$.

Let the vector-valued function $f: I \rightarrow D$ in Proposition 2.1 be written as $f(x)=\left(f_{1}(x)\right.$, $\left.f_{2}(x)\right)$. The required CHFIF is now defined as follows.

Definition 2.2. Suppose $\left\{\left(x, f_{1}(x)\right): x \in I\right\}$ is the projection of the attractor $G$ (cf. (2.5)) on $\mathbb{R}^{2}$. Then, the function $f_{1}(x)$ is called a coalescence hidden variable FIF (CHFIF) for the given interpolation data $\left\{\left(x_{n}, y_{n}\right) \mid n=0,1, \ldots, N\right\}$.

Proposition 2.1 gives that the graph of the vector-valued function $f(x)$ is the attractor of the IFS given by (2.4) if and only if the fixed point $f$ of the Read-Bajraktarević operator 
$T$ on the space of continuous vector-valued functions from $I$ to $D$ satisfies

$$
T f(x)=f(x)=F_{n}\left(L_{n}^{-1}(x), f\left(L_{n}^{-1}(x)\right)\right), \quad x \in I_{n}, n=1,2, \ldots, N .
$$

The image $T f$ of the vector-valued function $f$ can be written componentwise as $\left(T_{1} f_{1}\right.$, $T_{2} f_{2}$ ), where $T_{1}$ and $T_{2}$ are the componentwise Read-Bajraktarević operators from $I$ to $\mathbb{R}$. Thus, CHFIFs satisfy the following functional equation for all $x \in I$ :

$$
T_{1} f_{1}\left(L_{n}(x)\right)=f_{1}\left(L_{n}(x)\right)=F_{n}^{1}\left(x, f_{1}(x), f_{2}(x)\right)=\alpha_{n} f_{1}(x)+\beta_{n} f_{2}(x)+p_{n}(x) .
$$

Similarly, the projection $\left\{\left(x, f_{2}(x)\right): x \in I\right\}$ of the attractor $G$ is self-affine in nature. The fractal function $f_{2}(x)$ interpolates the data $\left\{\left(x_{n}, z_{n}\right): n=0,1, \ldots, N\right\}$ and satisfies the functional equation for all $x \in I$,

$$
T_{2} f_{2}\left(L_{n}(x)\right)=f_{2}\left(L_{n}(x)\right)=F_{n}^{2}\left(x, f_{2}(x)\right)=\gamma_{n} f_{2}(x)+q_{n}(x) .
$$

Since the graph of $f_{1}$ is a projection of $G$, it need not be union of its affine transformations. Hence, CHFIFs are generally non-self-affine in nature. If $y_{n}=z_{n}$ and $\alpha_{n}+\beta_{n}=\gamma_{n}$, CHFIF $f_{1}(x)$ coincides with the self-affine fractal function $f_{2}(x)$ for the same interpolation data. Hence, the CHFIF from a nondiagonal IFS is self-affine in this case. Also, if $\beta_{n}=0$, for $n=1,2, \ldots, N$, then the CHFIF $f_{1}$ is obviously always self-affine. It is observed that [9] depending on the parameter of nondiagonal IFS (cf. (2.4)), the modulus of continuity of $f_{1}$ is $O\left(|t|^{\mu}(\log t)^{n}\right)$, where $n=0,1$, or 2 .

\section{Calculus of vector-valued $C^{1}$-FIF}

First, it is shown that the integral of vector-valued FIF $f$ is also a vector-valued FIF to a different set of interpolation data if any of the values $\left(\hat{y}_{0}, \hat{z}_{0}\right)$ or $\left(\hat{y}_{N}, \hat{z}_{N}\right)$ of the integral of $f$ is known at an endpoint of the interval. Set $\hat{y}_{N}=\hat{y}_{0}+\left(\sum_{i=1}^{N} a_{i}\left\{\beta_{i}\left(\hat{z}_{N}-\right.\right.\right.$ $\left.\left.\left.\hat{z}_{0}\right)+\int_{x_{0}}^{x_{N}} p_{i}(\tau) d \tau\right\}\right) /\left(1-\sum_{i=1}^{N} a_{i} \alpha_{i}\right), \hat{z}_{N}=\hat{z}_{0}+\left(\sum_{i=1}^{N} a_{i} \int_{x_{0}}^{x_{N}} q_{i}(\tau) d \tau\right) /\left(1-\sum_{i=1}^{N} a_{i} \gamma_{i}\right), \hat{p}_{n}(x)$ $=\hat{y}_{n-1}-a_{n}\left(\alpha_{n} \hat{y}_{0}+\beta_{n} \hat{z}_{0}\right)+a_{n} \int_{x_{0}}^{x} p_{n}(\tau) d \tau, \hat{q}_{n}(x)=\hat{z}_{n-1}-a_{n} \gamma_{n} \hat{z}_{0}+a_{n} \int_{x_{0}}^{x} q_{n}(\tau) d \tau$, and

$$
\begin{gathered}
\hat{y}_{n}=\hat{y}_{0}+\sum_{i=1}^{n} a_{i}\left\{\alpha_{i}\left(\hat{y}_{N}-\hat{y}_{0}\right)+\beta_{i}\left(\hat{z}_{N}-\hat{z}_{0}\right)+\int_{x_{0}}^{x_{N}} p_{i}(\tau) d \tau\right\}, \\
\hat{z}_{n}=\hat{z}_{0}+\sum_{i=1}^{n} a_{i}\left\{\gamma_{i}\left(\hat{z}_{N}-\hat{z}_{0}\right)+\int_{x_{0}}^{x_{N}} q_{i}(\tau) d \tau\right\}, \\
\hat{F}_{n}(x, y, z)=\left(\hat{F}_{n}^{1}(x, y, z), \hat{F}_{n}^{2}(x, z)\right),
\end{gathered}
$$

where $\hat{F}_{n}^{1}(x, y, z)=a_{n}\left(\alpha_{n} y+\beta_{n} z\right)+\hat{p}_{n}(x), \hat{F}_{n}^{2}(x, z)=a_{n} \gamma_{n} z+\hat{q}_{n}(x)$.

Proposition 3.1. Let $f$ be the vector-valued FIF associated with $\left\{\left(L_{n}(x), F_{n}(x, y, z)\right) ; n=\right.$ $1,2, \ldots, N\}$, where $F_{n}$ is defined by (2.3). Let

$$
\widehat{f}_{1}(x)=\hat{y}_{0}+\int_{x_{0}}^{x} f_{1}(\tau) d \tau, \quad \widehat{f}_{2}(x)=\widehat{z}_{0}+\int_{x_{0}}^{x} f_{2}(\tau) d \tau
$$


then $\hat{f}=\left(\hat{f}_{1}, \hat{f}_{2}\right)$ is the vector-valued FIF associated with $\left\{\left(L_{n}(x), \hat{F}_{n}(x, y, z)\right) ; n=1,2, \ldots, N\right\}$ such that $\hat{f}\left(x_{n}\right)=\left(\hat{y}_{n}, \hat{z}_{n}\right)$ for $n=0,1,2, \ldots, N$, where $\hat{y}_{n}, \hat{z}_{n}$, and $\hat{F}_{n}^{1}(x, y, z)$ are defined by (3.1)-(3.3), respectively.

Proof. Using the definition of $\hat{f}_{1}$ for $x \in I$,

$$
\begin{aligned}
\hat{f}_{1}\left(L_{n}(x)\right) & =\hat{y}_{0}+\int_{x_{0}}^{L_{n}(x)} f_{1}(\tau) d \tau=\hat{y}_{0}+\int_{x_{0}}^{x_{n-1}} f_{1}(\tau) d \tau+\int_{x_{n-1}}^{L_{n}(x)} f_{1}(\tau) d \tau \\
& =\hat{f}_{1}\left(x_{n-1}\right)+a_{n} \int_{x_{0}}^{x} f_{1}\left(L_{n}(\tau)\right) d \tau .
\end{aligned}
$$

So, using (2.7),

$$
\begin{aligned}
\hat{f}_{1}\left(L_{n}(x)\right) & =\hat{y}_{n-1}+a_{n} \int_{x_{0}}^{x}\left(\alpha_{n} f_{1}(\tau)+\beta_{n} f_{2}(\tau)+p_{n}(\tau)\right) d \tau \\
& =\hat{y}_{n-1}+a_{n}\left[\alpha_{n}\left(\hat{f}_{1}(x)-\hat{y}_{0}\right)+\beta_{n}\left(\hat{f}_{2}(x)-\hat{z}_{0}\right)\right]+a_{n} \int_{x_{0}}^{x} p_{n}(\tau) d \tau
\end{aligned}
$$

In view of (2.7) and (3.6), the functional equation for $\hat{f}_{1}$ can be defined as follows:

$$
\hat{F}_{n}^{1}(x, y, z)=\hat{f}_{1}\left(L_{n}(x)\right)=a_{n}\left(\alpha_{n} y+\beta_{n} z\right)+\hat{p}_{n}(x)
$$

Similarly, using (2.8), the functional equation for $\hat{f}_{2}$ can be defined as

$$
\hat{F}_{n}^{2}(x, y, z)=\hat{f}_{2}\left(L_{n}(x)\right)=a_{n} \gamma_{n} z+\hat{q}_{n}(x)
$$

Thus, $\hat{f}_{1}$ is the CHFIF associated with $\left\{\left(L_{n}(x), \hat{F}_{n}(x, y, z)\right) ; n=1,2, \ldots, N\right\}$ to a different set of interpolation data. Since $\hat{f}_{1}$ and $\hat{f}_{2}$ are continuous functions, the continuity conditions are valid at the new interpolation points.

The functional values for the CHFIF $\hat{f}_{1}$ are obtained by putting $x=x_{N}$ in (3.6). Hence,

$$
\hat{y}_{n}=\hat{y}_{n-1}+a_{n}\left[\alpha_{n}\left(\hat{y}_{N}-\hat{y}_{0}\right)+\beta_{n}\left(\hat{z}_{N}-\hat{z}_{0}\right)\right]+a_{n} \int_{x_{0}}^{x_{N}} p_{n}(\tau) d \tau
$$

Inductively, using this equation up to $n=1$, (3.1) follows. Putting $n=N$ in (3.1) and solving for $\hat{y}_{N}$ give the relation between $\hat{y}_{N}$ and $\hat{y}_{0}$. Similarly, the functional values for the FIF $\hat{f}_{2}$ can be obtained by simplifying the expression for $\hat{f}_{2}\left(L_{n}(x)\right)$ and are omitted.

Remark 3.2. If the value of integral of the vector-valued FIF is known at the final endpoint $x_{N}$ instead of the initial endpoint, an analogue of Proposition 3.1 can be found as follows. Define

$$
\hat{f}_{1}(x)=\hat{y}_{N}-\int_{x}^{x_{N}} f_{1}(\tau) d \tau, \quad \hat{f}_{2}(x)=\widehat{z}_{N}-\int_{x}^{x_{N}} f_{2}(\tau) d \tau
$$


then $\hat{f}=\left(\hat{f}_{1}, \hat{f}_{2}\right)$ is the vector-valued FIF associated with $\left\{\left(L_{n}(x), \hat{F}_{n}(x, y, z)\right) ; n=1,2, \ldots\right.$, $N\}$, where $\hat{F}_{n}(x, y, z)=\left(\hat{F}_{n}^{1}(x, y, z), \hat{F}_{n}^{2}(x, z)\right), \hat{F}_{n}^{1}(x, y, z)=a_{n}\left(\alpha_{n} y+\beta_{n} z\right)+\hat{p}_{n}(x), \hat{F}_{n}^{2}(x, z)=$ $a_{n} \gamma_{n} z+\hat{q}_{n}(x), \quad \hat{p}_{n}(x)=\hat{y}_{n}-a_{n}\left(\alpha_{n} \hat{y}_{N}+\beta_{n} \hat{z}_{N}\right)-a_{n} \int_{x}^{x_{N}} p_{n}(\tau) d \tau, \quad \hat{y}_{n}=\hat{y}_{N}+$ $\sum_{i=n+1}^{N} a_{i}\left\{\alpha_{i}\left(\hat{y}_{N}-\hat{y}_{0}\right)+\beta_{i}\left(\hat{z}_{N}-\hat{z}_{0}\right)+\int_{x_{0}}^{x_{N}} p_{i}(\tau) d \tau\right\}, \hat{q}_{n}(x)=\hat{z}_{n}-a_{n} \alpha_{n} \hat{z}_{N}-a_{n} \int_{x}^{x_{N}} q_{n}(\tau) d \tau$, $\hat{z}_{n}=\hat{z}_{N}-\sum_{i=n+1}^{N} a_{i}\left\{\alpha_{i}\left(\hat{z}_{N}-\hat{z}_{0}\right)+\int_{x_{0}}^{x_{N}} q_{i}(\tau) d \tau\right\}, \hat{y}_{0}$ and $\hat{y}_{N}, \hat{z}_{0}$ and $\hat{z}_{N}$ are related in same manner as in Proposition 3.1.

Using Proposition 3.1 and Remark 3.2, a relation between a vector-valued FIF and its primitive is found through their IFS in the following proposition.

Proposition 3.3. Let $f$ and $\hat{f}$ be the vector-valued FIFs defined in Proposition 3.1 or Remark 3.2. Then, $f$ is a primitive of $\hat{f}$ if and only if $\hat{f}$ is the vector-valued FIF associated with $\left\{\left(L_{n}(x), \widehat{F}_{n}(x, y, z)\right) ; n=1,2, \ldots, N\right\}$, where for $n=1,2, \ldots, N$,

$$
\begin{gathered}
\hat{F}_{n}(x, y, z)=\left(\hat{F}_{n}^{1}(x, y, z), \hat{F}_{n}^{2}(x, z)\right), \\
\hat{F}_{n}^{1}(x, y, z)=\hat{\alpha}_{n} y+\hat{\beta}_{n} z+\hat{p}_{n}(x), \quad \hat{F}_{n}^{2}(x, z)=\hat{\gamma}_{n} z+\hat{q}_{n}(x), \\
\frac{\hat{\alpha}_{n}}{a_{n}}=\alpha_{n}, \quad \frac{\hat{\beta}_{n}}{a_{n}}=\beta_{n}, \quad \frac{\hat{\gamma}_{n}}{a_{n}}=\gamma_{n}, \quad \hat{p}_{n}^{\prime}=a_{n} p_{n}, \quad \hat{q}_{n}^{\prime}=a_{n} q_{n} .
\end{gathered}
$$

Proof. The necessary part is a direct consequence of Proposition 3.1 and Remark 3.2 by using the fundamental theorem of Calculus. For sufficiency, the join-up conditions of the IFS give the following relations:

$$
\begin{aligned}
\hat{y}_{n}-\hat{y}_{n-1} & =\hat{F}_{n}^{1}\left(x_{N}, \hat{y}_{N}, \hat{z}_{N}\right)-\hat{F}_{n}^{1}\left(x_{0}, \hat{y}_{0}, \hat{z}_{0}\right) \\
& =\hat{\alpha}_{n}\left(\hat{y}_{N}-\hat{y}_{0}\right)+\hat{\beta}_{n}\left(\hat{z}_{N}-\hat{z}_{0}\right)+\hat{p}_{n}\left(x_{N}\right)-\hat{p}_{n}\left(x_{0}\right) \\
& =a_{n}\left[\alpha_{n}\left(\hat{y}_{N}-\hat{y}_{0}\right)+\beta_{n}\left(\hat{z}_{N}-\hat{z}_{0}\right)\right]+a_{n} \int_{x_{0}}^{x_{N}} p_{n}(\tau) d \tau .
\end{aligned}
$$

Since both values of $\hat{y}_{N}$ and $\hat{y}_{0}$ are known if any one of these is given, the above relation inductively generates the interpolation values $\hat{y}_{n}$ of $\hat{f}_{1}$ as $\hat{y}_{n}=\hat{y}_{0}-\sum_{i=1}^{n}\left(\hat{y}_{i}-\hat{y}_{i-1}\right)=\hat{y}_{0}-$ $\sum_{i=1}^{n} a_{i}\left\{\alpha_{i}\left(\hat{y}_{N}-\hat{y}_{0}\right)+\beta_{i}\left(\hat{z}_{N}-\widehat{z}_{0}\right)+\int_{x_{0}}^{x_{N}} p_{i}(\tau) d \tau\right\}, n=1, \ldots, N-1$.

Further, the condition $\hat{p}_{n}^{\prime}=a_{n} p_{n}$ gives $\hat{p}_{n}(x)=a_{n} \int_{x_{0}}^{x} p_{n}+H_{n}$ for some constant $H_{n}$. The constant $H_{n}$, uniquely determined by the join-up condition $\hat{F}_{n}\left(x_{0}, \hat{y}_{0}, \hat{z}_{0}\right)=\left(\hat{y}_{n-1}\right.$, $\left.\hat{z}_{n-1}\right)$, is given by $H_{n}=\hat{y}_{n-1}-a_{n} \alpha_{n} \hat{y}_{0}-a_{n} \beta_{n} \hat{z}_{0}$. Thus, $\hat{p}_{n}$ is also uniquely determined as $\hat{p}_{n}(x)=\hat{y}_{n-1}-a_{n}\left(\alpha_{n} \hat{y}_{0}+\beta_{n} \hat{z}_{0}\right)+a_{n} \int_{x_{0}}^{x} p_{n}$. Since the expressions for $\hat{y}_{n}$ and $\hat{p}_{n}$ are the same as in Proposition 3.1, it follows that ${\hat{f}_{1}^{\prime}}^{\prime}=f_{1}$. Similarly, using the relation between $\hat{z}_{n}$ and $\hat{z}_{n-1}$, the join-up condition, and Proposition 3.1, $\hat{f}_{2}^{\prime}=f_{2}$. Analogously, if $\hat{f}$ is defined as in Remark 3.2, it can be seen that $\hat{f}^{\prime}=f$.

Remark 3.4. In the construction of $\hat{f}$ from the given vector-valued FIF $f$ obtained in Proposition 3.1 and Remark 3.2, the value of $\hat{f}$ is assumed to be known at one of the 
endpoints of the interval $I$. Hence, using Proposition 3.1 and Remark 3.2, a twice differentiable vector-valued FIF can be constructed when values of $\hat{f}$ and its integral are known at any combination of the endpoints of the interval $I$. In general, using Proposition 3.1 and Remark 3.2, we can construct a vector-valued $C^{r}$-FIF that interpolates a different data, when the values of each of $r$ successive integrals of a vector-valued FIF are prescribed at any combination of endpoints.

\section{Construction of spline CHFIF}

The construction of spline CHFIF or $C^{r}$-CHFIF that interpolates the prescribed data is found as the fixed point of a suitably chosen IFS and the parameters of such an IFS are determined by finding the solution of a system of equations in which any type of boundary conditions can be considered.

Let $\left\{\left(x_{0}, y_{0}\right),\left(x_{1}, y_{1}\right), \ldots,\left(x_{N}, y_{N}\right)\right\}, x_{0}<x_{1}<\cdots<x_{N}$, be the given data points and it is extended to generalized data by providing the real parameters $z_{0}, z_{1}, \ldots, z_{N}$. Let $G^{r}=\{g \in$ $C^{r}\left(I, \mathbb{R}^{2}\right) \mid g\left(x_{0}\right)=\left(y_{0}, z_{0}\right)$ and $\left.g\left(x_{N}\right)=\left(y_{N}, z_{N}\right)\right\}$, where $r$ is some nonnegative integer. Let $d^{*}$ be the $C^{r}$-norm on $\mathscr{G}^{r}$. For $x \in I_{n}, n=1,2, \ldots, N$, define the Read-Bajraktarević operator $T$ on the space $\left(\mathscr{G}^{r}, d^{*}\right)$ of vector-valued functions by

$$
\begin{aligned}
\operatorname{Tg}(x) & =F_{n}\left(L_{n}^{-1}(x), g\left(L_{n}^{-1}(x)\right)\right) \\
& =\left(\alpha_{n} g_{1}\left(L_{n}^{-1}(x)\right)+\beta_{n} g_{2}\left(L_{n}^{-1}(x)\right)+p_{n}(x), \gamma_{n} g_{2}\left(L_{n}^{-1}(x)\right)+q_{n}(x)\right),
\end{aligned}
$$

where $L_{n}(x)=a_{n} x+b_{n}$ satisfies (2.1), $p_{n}(x), q_{n}(x)$ are suitably chosen polynomials, $\left|\alpha_{n}\right|<$ $a_{n}^{r}$, and $\left|\beta_{n}\right|+\left|\gamma_{n}\right|<a_{n}^{r}$ for $n=1,2, \ldots, N$. The restrictions on free variables and constrained free variables imply that $T$ is a contractive operator on $\left(G^{r}, d^{*}\right)$. Since $\left(G^{r}, d^{*}\right)$ is complete, the fixed point $f=\left(f_{1}, f_{2}\right)$ of $T$ satisfies the functional relations, $f_{1}\left(L_{n}(x)\right)=$ $\alpha_{n} f_{1}(x)+\beta_{n} f_{2}(x)+p_{n}(x)$ and $f_{2}\left(L_{n}(x)\right)=\gamma_{n} f_{2}(x)+q_{n}(x)$ for $n=1,2, \ldots, N$. In general, $f_{1}$ interpolates data different from the prescribed data. Using Proposition 3.3, it follows that $f_{1}^{\prime}$ satisfies the functional relation

$$
f_{1}^{\prime}\left(L_{n}(x)\right)=\frac{\alpha_{n} f_{1}^{\prime}(x)+\beta_{n} f_{2}^{\prime}(x)+p_{n}^{\prime}(x)}{a_{n}}, \quad n=1,2, \ldots, N
$$

Since $\left|\alpha_{n}\right| / a_{n} \leq\left|\alpha_{n}\right| / a_{n}^{r}<1$ and $\left(\left|\beta_{n}\right|+\left|\gamma_{n}\right|\right) / a_{n} \leq\left(\left|\beta_{n}\right|+\left|\gamma_{n}\right|\right) / a_{n}^{r}<1, f_{1}^{\prime}$ is a CHFIF for different data. Inductively, using the above arguments, the following relations are obtained:

$$
f_{1}^{(k)}\left(L_{n}(x)\right)=\frac{\alpha_{n} f_{1}^{(k)}(x)+\beta_{n} f_{2}^{(k)}(x)+p_{n}^{(k)}(x)}{a_{n}^{k}}, \quad n=1,2, \ldots, N ; k=0,1,2, \ldots, r,
$$

where $f_{1}^{(0)}=f_{1}, f_{2}^{(0)}=f_{2}$, and $p^{(0)}=p$. Since $\left|\alpha_{n}\right| / a_{n}^{k} \leq\left|\alpha_{n}\right| / a_{n}^{r}<1$ and $\left(\left|\beta_{n}\right|+\left|\gamma_{n}\right|\right) / a_{n}$ $\leq\left(\left|\beta_{n}\right|+\left|\gamma_{n}\right|\right) / a_{n}^{r}<1, f_{1}^{(k)}(k=2,3, \ldots, r)$ is a CHFIF for different data. In general, $f_{1}^{(k)}$ $(k=2,3, \ldots, r)$ interpolates data different from the prescribed data. In particular, $f_{1}^{(r)}$ is an affine CHFIF for different data, if the polynomial $p_{n}^{(r)}$ occurring in (4.3) with $k=r$ 
is affine. Thus, $p_{n}(x)$ is to be chosen as a polynomial of degree $(r+1)$ for $f_{1}$ to be a $C^{r}$-CHFIF. Let $p_{n}(x)=\sum_{k=0}^{r+1} p_{k n} x^{k}, n=1,2, \ldots, N$, where the coefficients $p_{k n}$ have to be determined suitably such that $f_{1}$ interpolates the prescribed data. The continuity of $f_{1}^{(k)}$ on $I$ gives

$$
f_{1}^{(k)}\left(L_{n+1}\left(x_{0}\right)\right)=f_{1}^{(k)}\left(L_{n}\left(x_{N}\right)\right), \quad k=0,1, \ldots, r ; n=1,2, \ldots, N-1 .
$$

Hence, using (4.3), the following $(r+1)(N-1)$ join-up conditions can be obtained for $k=0,1, \ldots, r$ and $n=1,2, \ldots, N-1$ :

$$
\frac{\alpha_{n+1} f_{1}^{(k)}\left(x_{0}\right)+\beta_{n+1} f_{2}^{(k)}\left(x_{0}\right)+p_{n+1}^{(k)}\left(x_{0}\right)}{a_{n+1}^{k}}=\frac{\alpha_{n} f_{1}^{(k)}\left(x_{N}\right)+\beta_{n} f_{2}^{(k)}\left(x_{N}\right)+p_{n}^{(k)}\left(x_{N}\right)}{a_{n}^{k}} .
$$

Also, (4.3) implies that at the endpoints on the interval, the values of $f_{1}^{(k)}$ satisfy the following $2 r$-conditions:

$$
\begin{gathered}
f_{1}^{(k)}\left(x_{0}\right)=\frac{\alpha_{1} f_{1}^{(k)}\left(x_{0}\right)+\beta_{1} f_{2}^{(k)}\left(x_{0}\right)+p_{1}^{(k)}\left(x_{0}\right)}{a_{1}^{k}}, \quad k=1,2, \ldots, r, \\
f_{1}^{(k)}\left(x_{N}\right)=\frac{\alpha_{N} f_{1}^{(k)}\left(x_{N}\right)+\beta_{N} f_{2}^{(k)}\left(x_{N}\right)+p_{N}^{(k)}\left(x_{N}\right)}{a_{N}^{k}}, \quad k=1,2, \ldots, r .
\end{gathered}
$$

Finally, the given interpolation conditions are

$$
f_{1}\left(x_{n}\right)=y_{n}, \quad n=0,1, \ldots, N
$$

Similarly, if $q_{n}(x)=\sum_{k=0}^{r+1} q_{k n} x^{k}, n=1,2, \ldots, N$, the analogous equations for the coefficients $q_{k n}$ associated with self-affine fractal function $f_{2}$ are

$$
\begin{gathered}
\frac{\gamma_{n+1} f_{2}^{(k)}\left(x_{0}\right)+q_{n+1}^{(k)}\left(x_{0}\right)}{a_{n+1}^{k}}=\frac{\gamma_{n} f_{2}^{(k)}\left(x_{N}\right)+q_{n}^{(k)}\left(x_{N}\right)}{a_{n}^{k}}, \quad k=0,1, \ldots, r ; n=1,2, \ldots, N-1, \\
f_{2}^{(k)}\left(x_{0}\right)=\frac{\gamma_{1} f_{2}^{(k)}\left(x_{0}\right)+q_{1}^{(k)}\left(x_{0}\right)}{a_{1}^{k}}, \quad k=1,2, \ldots, r, \\
f_{2}^{(k)}\left(x_{N}\right)=\frac{\gamma_{N} f_{2}^{(k)}\left(x_{N}\right)+q_{N}^{(k)}\left(x_{N}\right)}{a_{N}^{k}}, \quad k=1,2, \ldots, r, \\
f_{2}\left(x_{n}\right)=z_{n}, \quad n=0,1, \ldots, N .
\end{gathered}
$$

The set of (4.8) involves $(r+1)(N-1)+2 r+(N+1)=(r+2) N+r$ conditions and $(r+2) N+2 r$ number of unknowns (i.e., $f_{2}^{(k)}\left(x_{0}\right), f_{2}^{(k)}\left(x_{N}\right)$ for $k=1,2, \ldots, r$ and the coefficients of the polynomials $\left.q_{n}(x), q_{k n}, k=0,1, \ldots, r+1, n=1,2, \ldots, N\right)$. Therefore, assuming $r$ suitable relations involving the values of $C^{r}$-fractal function $f_{2}$ or the values of its derivatives at endpoints of $\left[x_{0}, x_{N}\right]$, the values of $f_{2}^{(k)}\left(x_{0}\right)$ and $f_{2}^{(k)}\left(x_{N}\right)$ for 
$k=1,2, \ldots, r$ can be obtained from (4.8). Now, from (4.5)-(4.7), the total number of the conditions for $f_{1}$ to interpolate the prescribed data is $(r+2) N+r$. Equations (4.5)-(4.7) involve $2 r$ unknowns: $f_{1}^{(k)}\left(x_{0}\right)$ and $f_{1}^{(k)}\left(x_{N}\right)$ for $k=1,2, \ldots, r$ and $(r+2) N$ unknown coefficients, $p_{k n}, k=0,1, \ldots, r+1, n=1,2, \ldots, N$ of the polynomials $p_{n}(x)$. Consequently, in all $(r+2) N+2 r$ number of unknowns are to be determined. The principle of construction of spline or $C^{r}$-CHFIF is to determine the above unknowns by choosing additional suitable $r$-restrictions on the values of $C^{r}$-CHFIF or the values of its derivatives at the boundary points of $\left[x_{0}, x_{N}\right]$ such that (4.5)-(4.7) together with these additional conditions are linearly independent. These unknowns are determined uniquely as solutions of these linearly independent systems of equations. The existence of the attractor for a $C^{r}$-CHFIF is guaranteed by the fixed point theorem and the assumptions $\left|\alpha_{n}\right|<a_{n}^{r}$ and $\left|\beta_{n}\right|+\left|\gamma_{n}\right|<a_{n}^{r}$. Hence, the required $C^{r}$-CHFIF $f_{1}$ interpolating the prescribed data is constructed as the attractor of the following IFS given by

$$
\left\{\mathbb{R}^{3} ; \omega_{n}(x, y)=\left(L_{n}(x), F_{n}(x, y, z)\right)=\left(\alpha_{n} y+\beta_{n} z+p_{n}(x), \gamma_{n} z+q_{n}(x)\right), n=1,2, \ldots, N\right\},
$$

where $\left|\alpha_{n}\right|<a_{n}^{r},\left|\beta_{n}\right|+\left|\gamma_{n}\right|<a_{n}^{r}, q_{n}(x)$, and $p_{n}(x), n=1,2, \ldots, N$, are the polynomials with coefficients $q_{k n}$ and $p_{k n}$, respectively, computed by solving the linear independent system of equations, given by the above procedure. The flexibility of these choices of boundary conditions allows for the construction of a wide range of spline CHFIFs that may be self-affine or non-self-affine in nature. In such a construction, for a given choice of boundary conditions, depending upon the nature of the problem or simply the discretion of the user, an infinite number of suitable spline CHFIFs may be constructed due to the freedom of choices in the free variables $\alpha_{n}, \gamma_{n}$, constrained free variables $\beta_{n}$ for $n=1,2, \ldots, N$, the free parameters $z_{n}, n=0,1,2, \ldots, N$, and the boundary conditions of the self-affine fractal function $f_{2}$.

\section{Examples of spline CHFIF}

We computationally generate examples of spline CHFIF $f_{1} \in C^{2}[0,1]$ from the IFS given by (4.9). The interpolation data set is taken as $\{(0,0),(2 / 5,1),(3 / 4,-1),(1,2)\}$ for spline CHFIF with $\alpha_{n} / a_{n}^{2}=0.8, n=1,2,3$. The interpolation data is extended to generalized interpolation data with the addition of hidden variables $z_{0}=3, z_{1}=2, z_{2}=8, z_{3}=5$ and $\gamma_{1} / a_{1}^{2}=0.3, \gamma_{2} / a_{2}^{2}=0.35, \gamma_{3} / a_{3}^{2}=0.4$. Suppose that the constrained free variables are chosen as $\beta_{1} / a_{1}^{2}=0.4, \beta_{2} / a_{2}^{2}=0.6, \beta_{3} / a_{3}^{2}=0.5$ for a spline CHFIF (Figure 5.1). By using (2.1), $L_{1}(x)=(2 / 5) x, L_{2}(x)=(7 / 20 x)+2 / 5$, and $L_{3}(x)=(1 / 4 x)+3 / 4$ in the IFS for our examples.

First, we calculate the polynomial $q_{n}(x)=\sum_{k=0}^{3} q_{k n} x^{k}$ for $n=1,2,3$ for our first 4 examples. By the principle of construction of spline CHFIF, we choose $f_{2}^{\prime}\left(x_{0}\right)=10$ and $f_{2}^{\prime}\left(x_{3}\right)=1$ in order to solve system of $(4.8)$. The computed $F_{n}^{2}(x, z)$ by using polynomials $q_{n}(x)$ are given in Table 5.1. For constructing the first example of spline CHFIF, we choose $f_{1}^{\prime}\left(x_{0}\right)=2$ and $f_{1}^{\prime}\left(x_{N}\right)=5$. With these choices, the system of (4.6)-(4.7) is solved to get the values of $p_{k n}$ in $p_{n}(x)=\sum_{k=0}^{3} p_{k n} x^{k}$ for $n=1,2,3$. Using $F_{n}^{1}(x, y, z)$ (Table 5.2) in the IFS (4.9), the desired spline CHFIF is generated (Figure 5.1). 


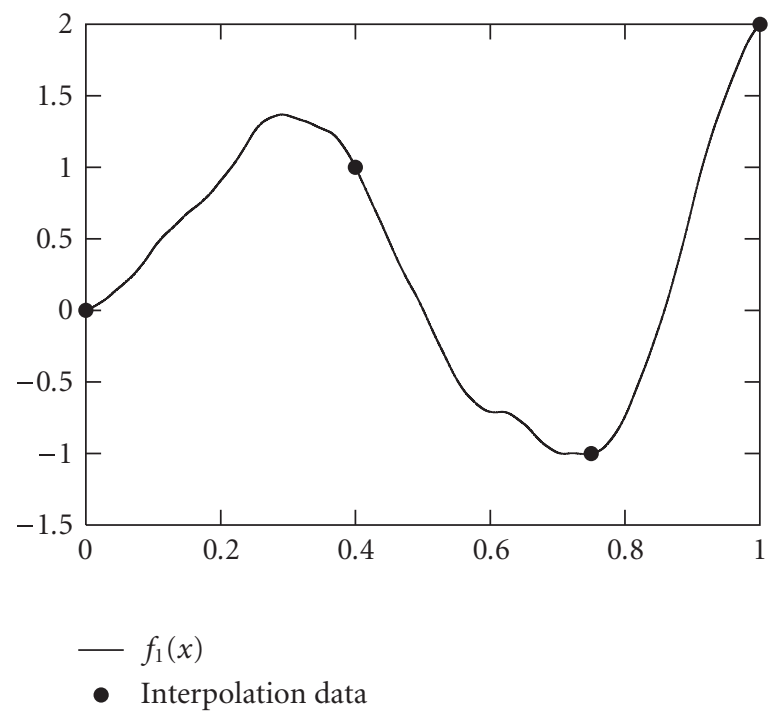

Figure 5.1. Spline CHFIF $f_{1}(x)$ for comparison.

Next, to construct an example of spline CHFIF with analogous boundary conditions as by Barnsley and Harrington [4], we choose $f_{1}^{\prime}\left(x_{0}\right)=2$ and $f_{1}^{\prime \prime}\left(x_{0}\right)=5$. By the principle of construction of CHFIF, the coefficients of $p_{n}(x)$ are computed (Table 5.2). The iteration of the resulting IFS code (4.9) generates the spline CHFIF (Figure 5.2). Similarly, Figures 5.3 and 5.4 are generated to see the effect of change in free variables $\alpha_{n}$ and constrained free variables $\beta_{n}$, respectively, on the shape of spline CHFIF in comparison to that given by Figure 5.1 with the same choice of two additional conditions, $f_{2}{ }^{\prime}\left(x_{0}\right)=10$ and $f_{2}{ }^{\prime}\left(x_{3}\right)=1$. In Figure 5.3, we have chosen $\alpha_{n} / a_{n}^{2}=-0.9$ for $n=1,3$ and $\alpha_{2} / a_{2}^{2}=0.9$. In Figure 5.4, we have chosen $\beta_{1} / a_{1}^{2}=-0.6, \beta_{2} / a_{2}^{2}=-0.6$ and $\beta_{3} / a_{3}^{2}=0.2$.

Next, we change the hidden variable $\gamma_{n}$ as $\gamma_{1} / a_{1}^{2}=-0.5, \gamma_{2} / a_{2}^{2}=0.3$, and $\gamma_{3} / a_{3}^{2}=-0.4$. Subsequently, with the same choice of boundary conditions $f_{2}^{\prime}\left(x_{0}\right)=10$ and $f_{2}\left(x_{3}\right)=1$, $F_{n}^{2}(x, z)$ is computed in Table 5.1. Using $f_{2}^{(k)}\left(x_{0}\right), f_{2}^{(k)}\left(x_{3}\right) ; k=1,2, F_{n}^{1}(x, y, z)$ (Table 5.2) is computed with $f_{1}^{\prime}\left(x_{0}\right)=2$ and $f_{1}^{\prime}\left(x_{3}\right)=5$ in order to see the effect of $\gamma_{n}$ on the shape of spline CHFIF (Figure 5.5) in comparison to that given by Figure 5.1. In Figure 5.6, we change only the hidden variable $z_{n}$ as $z_{0}=-7, z_{1}=-10, z_{2}=9, z_{3}=-8$ in order to compare with Figure 5.1.

In Figure 5.7, we change the boundary condition of spline fractal function $f_{2}(x)$ as $f_{2}{ }^{\prime \prime}\left(x_{0}\right)=10, f_{2}{ }^{\prime \prime}\left(x_{3}\right)=1$. Consequently, the fractal function $f_{2}$ changes and the effect of change in boundary conditions of $f_{2}$ on the shape of spline CHFIF (Figure 5.7) can be seen by comparing with Figure 5.1.

In all seven spline CHFIFs above, the second derivative $f_{1}^{\prime \prime}(x)$ is a non-self-affine in nature. Finally, we assume $y_{n}=z_{n}$ for $n=0,1,2,3, \alpha_{n}+\beta_{n}=\gamma_{n}$ for $n=1,2,3$ with the same boundary conditions $f_{1}^{\prime}\left(x_{0}\right)=f_{2}^{\prime}\left(x_{0}\right)=2$ and $f_{1}^{\prime}\left(x_{3}\right)=f_{2}^{\prime}\left(x_{3}\right)=5$. Here, we have considered $\gamma_{n} / a_{n}^{2}=0.8$ for $n=1,2,3$ and $\alpha_{1} / a_{1}^{2}=0.5, \alpha_{2} / a_{2}^{2}=0.4, \alpha_{3} / a_{3}^{2}=0.3, \beta_{1} / a_{1}^{2}=0.3$, 
Table 5.1. $F_{n}^{2}(x, z)$ used in the construction of $f_{1}(x)$.

\begin{tabular}{l|l}
\hline \multirow{2}{*}{ Figures 5.1-5.4 } & $F_{1}^{2}(x, z)=0.048 z+7.1155 x^{3}-11.7315 x^{2}+3.52 x+2.856$ \\
& $F_{2}^{2}(x, z)=0.0429 z-13.9198 x^{3}+18.9927 x^{2}+0.8413 x+1.8714$ \\
& $F_{3}^{2}(x, z)=0.025 z+4.0109 x^{3}-4.7468 x^{2}-2.3141 x+7.925$ \\
\hline \multirow{2}{*}{ Figure 5.5 } & $F_{1}^{2}(x, z)=-0.08 z+11.882 x^{3}-17.522 x^{2}+4.8 x+3.24$ \\
& $F_{2}^{2}(x, y)=0.0367 z-8.7332 x^{3}+10.3705 x^{2}+4.2892 x+1.8898$ \\
& $F_{3}^{2}(x, y)=-0.025 z+5.6159 x^{3}-8.0068 x^{2}-0.5591 x+8.075$ \\
\hline Figure 5.6 & $F_{1}^{2}(x, z)=0.048 z+16.9824 x^{3}-23.4544 x^{2}+3.52 x-6.664$ \\
& $F_{2}^{2}(x, z)=0.0429 z-52.7327 x^{3}+65.5487 x^{2}+6.2269 x-9.6999$ \\
& $F_{3}^{2}(x, z)=0.025 z+19.0457 x^{3}-20.8914 x^{2}-15.1293 x+9.175$ \\
\hline Figure 5.7 & $F_{1}^{2}(x, z)=0.048 z+3.6899 x^{3}-0.7521 x^{2}-4.0339 x+2.856$ \\
& $F_{2}^{2}(x, z)=0.0429 z-5.9516 x^{3}+7.7897 x^{2}+4.0761 x+1.8714$ \\
& $F_{3}^{2}(x, z)=0.025 z+1.5994 x^{3}-5.2041 x^{2}+0.5547 x+7.925$ \\
\hline & $F_{1}^{2}(x, z)=0.128 z+1.446 x^{3}-1.246 x^{2}+0.544 x$ \\
& $F_{2}^{2}(x, z)=0.098 z+11.83 x^{3}-16.4813 x^{2}+2.4552 x+1$ \\
& $F_{3}^{2}(x, z)=0.05 z-0.9909 x^{3}+0.0817 x^{2}+3.8091 x-1$ \\
\hline
\end{tabular}

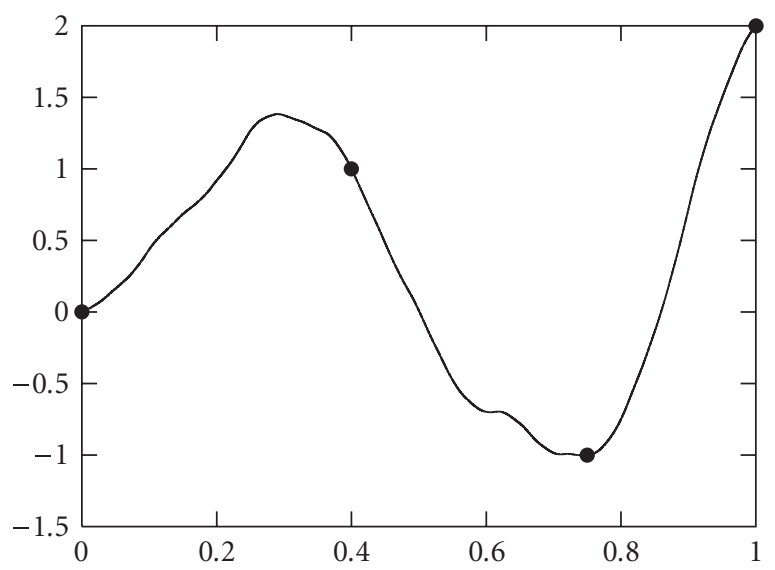

$-f_{1}(x)$

- Interpolation data

Figure 5.2. Effect on spline CHFIF by a change in boundary conditions of $f_{1}(x)$. 
12 Spline coalescence fractal interpolation functions

Table 5.2. $F_{n}^{1}(x, y, z)$ used in the construction of $f_{1}(x)$.

\begin{tabular}{|c|c|}
\hline Figure 5.1 & $\begin{array}{l}F_{1}^{1}(x, y, z)=0.128 y+0.064 z-5.9811 x^{3}+6.6931 x^{2}-0.096 x-0.192 \\
F_{2}^{1}(x, y, z)=0.098 y+0.0735 z-0.1352 x^{3}+2.1787 x^{2}-4.3865 x+0.7795 \\
F_{3}^{1}(x, y, z)=0.05 y+0.0313 z-5.0268 x^{3}+8.1848 x^{2}-0.3205 x-1.0938\end{array}$ \\
\hline Figure 5.2 & $\begin{array}{l}F_{1}^{1}(x, y, z)=0.128 y+0.064 z-1.7189 x^{3}+5.3755 x^{2}-3.0406 x-0.192 \\
F_{2}^{1}(x, y, z)=0.098 y+0.0735 z-14.524 x^{3}+21.7151 x^{2}-9.5341 x+0.7795 \\
F_{3}^{1}(x, y, z)=0.05 y+0.0313 z-2.5515 x^{3}+1.6161 x^{2}+3.7729 x-1.0938\end{array}$ \\
\hline Figure 5.3 & $\begin{array}{l}F_{1}^{1}(x, y, z)=-0.144 y+0.064 z-7.2999 x^{3}+8.0119 x^{2}+0.448 x-0.192 \\
F_{2}^{1}(x, y, z)=0.1102 y+0.0735 z-3.0304 x^{3}+6.9418 x^{2}-6.2789 x+0.7795 \\
F_{3}^{1}(x, y, z)=-0.0563 y+0.0313 z-5.4156 x^{3}+9.2811 x^{2}-0.8156 x-1.0938\end{array}$ \\
\hline Figure 5.4 & $\begin{array}{l}F_{1}^{1}(x, y, z)=0.128 y-0.096 z+6.2655 x^{3}-6.8335 x^{2}+1.504 x+0.288 \\
F_{2}^{1}(x, y, z)=0.098 y-0.0735 z+16.2854 x^{3}-25.1537 x^{2}+6.8193 x+1.2205 \\
F_{3}^{1}(x, y, z)=0.05 y+0.0125 z-0.8557 x^{3}-0.1762 x^{2}+3.9068 x-1.0375\end{array}$ \\
\hline Figure 5.5 & $\begin{array}{l}F_{1}^{1}(x, y, z)=0.128 y+0.064 z-2.8759 x^{3}+3.5879 x^{2}-0.096 x-0.192 \\
F_{2}^{1}(x, y, z)=0.098 y+0.0735 z+5.0471 x^{3}-5.7207 x^{2}-1.6694 x+0.7795 \\
F_{3}^{1}(x, y, z)=0.05 y+0.0313 z-3.2659 x^{3}+4.663 x^{2}+1.4404 x-1.0938\end{array}$ \\
\hline Figure 5.6 & $\begin{array}{l}F_{1}^{1}(x, y, z)=0.128 y+0.064 z-27.2463 x^{3}+28.1503 x^{2}-0.096 x+0.448 \\
F_{2}^{1}(x, y, z)=0.098 y+0.0735 z-36.6824 x^{3}+57.2174 x^{2}-22.6575 x+1.5145 \\
F_{3}^{1}(x, y, z)=0.05 y+0.0313 z-17.9536 x^{3}+33.9448 x^{2}-13.0599 x-0.7813\end{array}$ \\
\hline Figure 5.7 & $\begin{array}{l}F_{1}^{1}(x, y, z)=0.128 y+0.064 z+1.0682 x^{3}-1.8558 x^{2}+1.4035 x-0.192 \\
F_{2}^{1}(x, y, z)=0.098 y+0.0735 z+10.1125 x^{3}-13.3792 x^{2}+0.9237 x+0.7795 \\
F_{3}^{1}(x, y, z)=0.05 y+0.0313 z-1.2489 x^{3}+1.3367 x^{2}+2.7497 x-1.0938\end{array}$ \\
\hline Figure 5.8 & $\begin{array}{l}F_{1}^{1}(x, y, z)=0.08 y+0.048 z+1.446 x^{3}-1.246 x^{2}+0.544 x \\
F_{2}^{1}(x, y, z)=0.049 y+0.049 z+11.83 x^{3}-16.4813 x^{2}+2.4552 x+1 \\
F_{3}^{1}(x, y, z)=0.0187 y+0.0313 z-0.9909 x^{3}+0.0817 x^{2}+3.8091 x-1\end{array}$ \\
\hline
\end{tabular}

$\beta_{2} / a_{2}^{2}=0.4, \beta_{3} / a_{3}^{2}=0.5$. In this case, the projection of nondiagonal IFS code generates self-affine spline CHFIF (Figure 5.8). Thus, our approach offers great flexibility and diversity to an experimenter depending upon the need of the problem for the choice of a self-affine or non-self-affine spline FIF. 


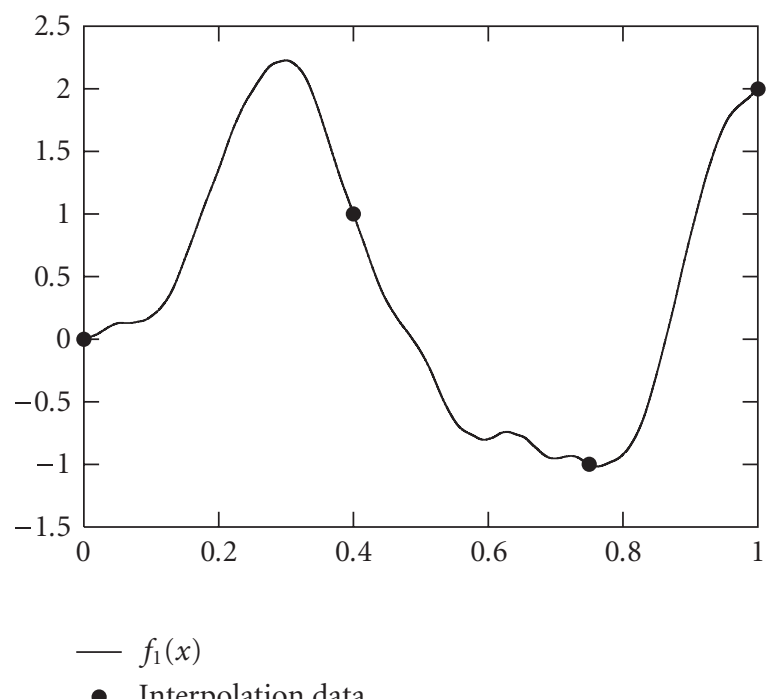

Figure 5.3. Effect on spline CHFIF by a change in the hidden variables $\alpha_{n}$.

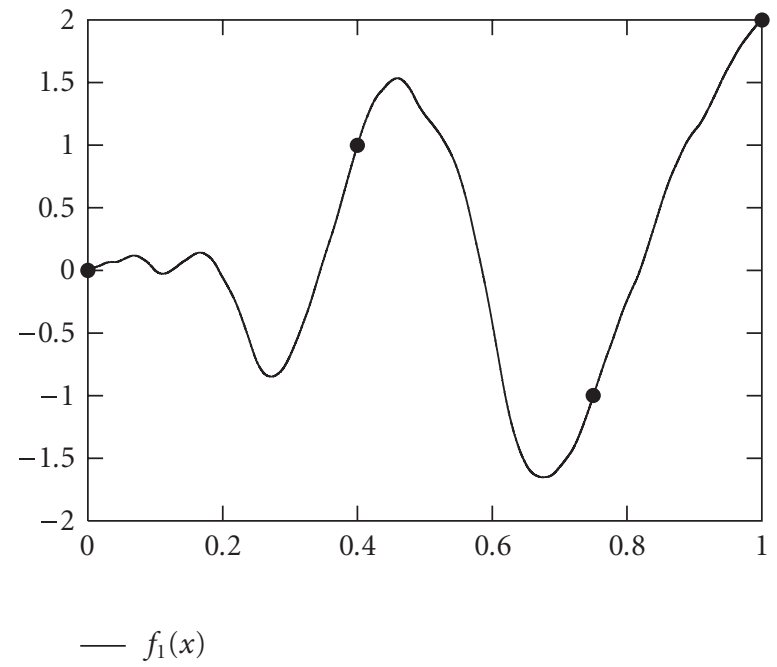

- Interpolation data

Figure 5.4. Effect on spline CHFIF by a change in the hidden variables $\beta_{n}$.

\section{Conclusion}

We have constructed the differentiable or spline CHFIFs for which certain derivative is a self-affine or non-self-affine fractal function depending on the nondiagonal IFS parameters. In our construction of a $C^{r}$-CHFIF $f_{1}$, we chose $\left|\alpha_{n}\right|<a_{n}^{r}$ and $\left|\beta_{n}\right|+\left|\gamma_{n}\right|<a_{n}^{r}$ for 
14 Spline coalescence fractal interpolation functions

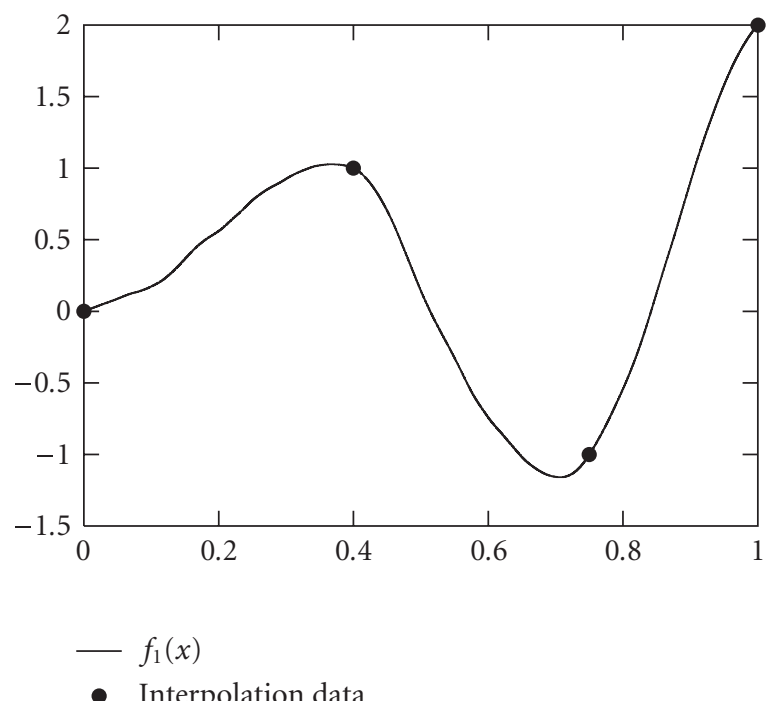

Figure 5.5. Effect on spline CHFIF by a change in the hidden variables $\gamma_{n}$.

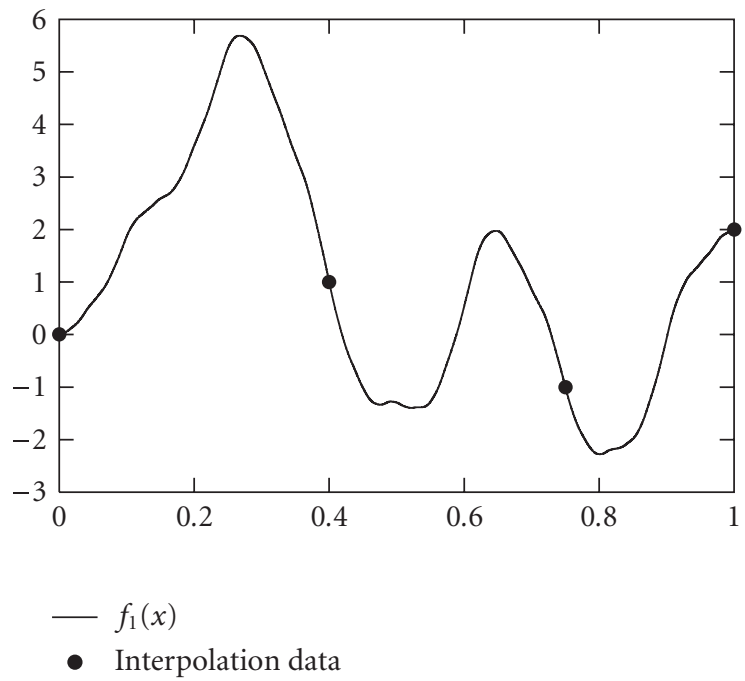

Figure 5.6. Effect on spline CHFIF by a change in the hidden variables $z_{n}$.

$n=1,2, \ldots, N$, where $a_{n}$ can be calculated from the given interpolation data. The system of (4.8) is solved with a suitable choice of boundary conditions for the coefficients of polynomial $q_{n}$ and the derivatives of the fractal function $f_{2}$ at endpoints. Finally, by prescribing the boundary conditions for the CHFIF, the coefficients of polynomials $p_{n}$ are 


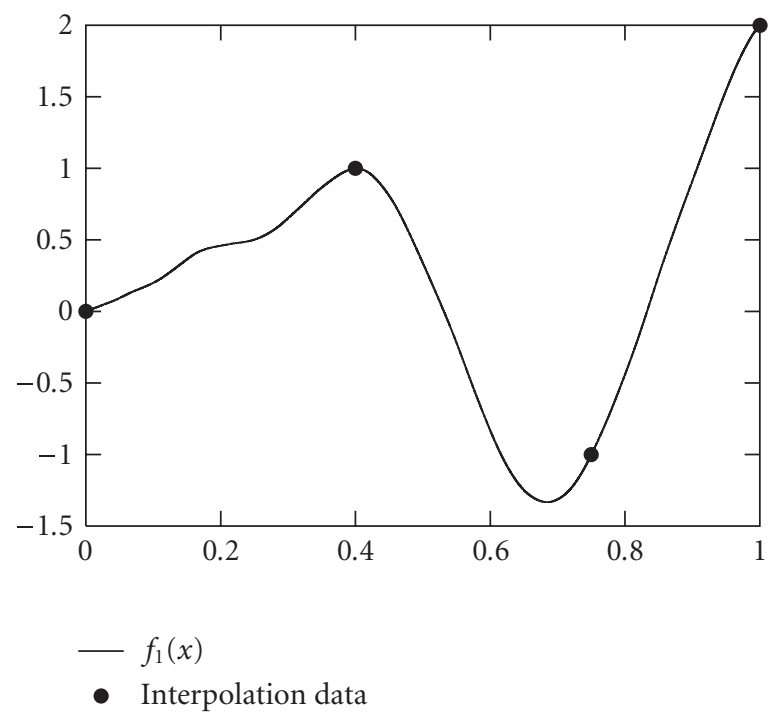

Figure 5.7. Effect on spline CHFIF by a change in the boundary conditions of $f_{2}(x)$.

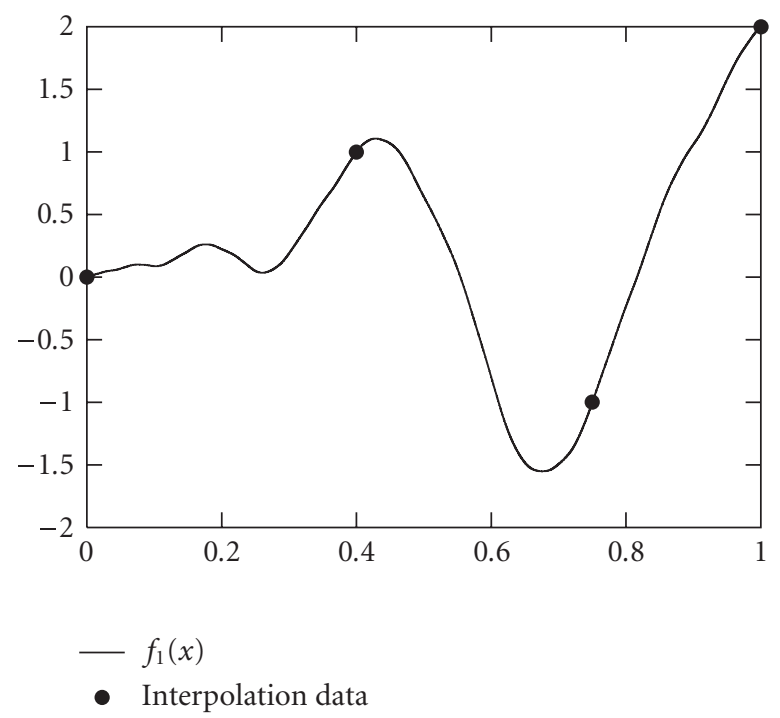

Figure 5.8. Self-affine spline CHFIF.

obtained from (4.5)-(4.7). The fixed-point theorem gives the existence of the attractor of the IFS given by (4.9) and the projection of the attractor is the desired $C^{r}$-CHFIF. Our construction allows admissibility of any kind of boundary conditions by using the solution of a system of equations. Our construction successfully answers the question "what 
sort of endpoint condition leads to unique solution" of Barnsley's and Harrington's [4]. Thus, the complex algebraic method for construction of a spline FIF [4] by using complicated matrices with a particular type of boundary conditions is no longer needed. The hidden variables, free variables, and constrained variables play an important role in determining the shape of differentiable CHFIF that is very useful in approximation theory. For given boundary conditions, an infinite number of spline CHFIF can be constructed interpolating the same data by varying the free variables $\alpha_{n}$, the constrained free variables $\beta_{n}$, the hidden variables $\gamma_{n}$, the free parameter $z_{n}$, or the boundary conditions of self-affine spline fractal function. It is felt that the spline CHFIF can be used in various scientific and engineering applications to capture the self-affine and non-self-affine nature simultaneously for relevant smooth objects.

\section{Acknowledgments}

The work was partially supported by the CSIR, India, Grant no. 9/92(160)/98-EMR-I. The authors are thankful to the referees for valuable suggestions.

\section{References}

[1] M. F. Barnsley, Fractal functions and interpolation, Constructive Approximation 2 (1986), no. 4, 303-329.

[2] _ Fractals Everywhere, Academic Press, Massachusetts, 1988.

[3] M. F. Barnsley, J. Elton, D. Hardin, and P. R. Massopust, Hidden variable fractal interpolation functions, SIAM Journal on Mathematical Analysis 20 (1989), no. 5, 1218-1242.

[4] M. F. Barnsley and A. N. Harrington, The calculus of fractal interpolation functions, Journal of Approximation Theory 57 (1989), no. 1, 14-34.

[5] M. F. Barnsley and L. P. Hurd, Fractal Image Compression, A K Peters, Massachusetts, 1993.

[6] A. K. B. Chand, A study on coalescence and spline fractal interpolation function, Ph.D. thesis, IIT Kanpur, Kanpur, 2005.

[7] A. K. B. Chand and G. P. Kapoor, Hidden variable bivariate fractal interpolation surfaces, Fractals 11 (2003), no. 3, 277-288.

[8] _ Generalized cubic spline fractal interpolation functions, SIAM Journal on Numerical Analysis 44 (2006), no. 2, 655-676.

[9] __ Coalescence hidden variable fractal interpolation functions and its smoothness analysis, preprint, 2005, http://arxiv.org/abs/math.DS/0511073.

[10] H. H. Hardy and R. A. Beier, Fractals in Reservoir Engineering, World Scientific, Singapore, 1994.

[11] J. E. Hutchinson, Fractals and self-similarity, Indiana University Mathematics Journal 30 (1981), no. $5,713-747$.

[12] B. B. Mandelbrot, The Fractal Geometry of Nature, W. H. Freeman, California, 1977.

[13] P. Maragos, Fractal aspects of speech signals: dimension and interpolation, International Conference on Acoustics, Speech, and Signal Processing (ICASSP '91), vol. 1, Ontario, 1991, pp. 417420.

[14] P. R. Massopust, Fractal Functions, Fractal Surfaces, and Wavelets, Academic Press, California, 1994.

[15] D. S. Mazel and M. H. Hayes, Using iterated function systems to model discrete sequences, IEEE Transactions on Signal Processing 40 (1992), no. 7, 1724-1734.

[16] M. A. Navascués, Fractal polynomial interpolation, Zeitschrift für Analysis und ihre Anwendungen 24 (2005), no. 2, 401-418. 
[17] M. A. Navascués and M. V. Sebastián, Some results of convergence of cubic spline fractal interpolation functions, Fractals 11 (2003), no. 1, 1-7.

[18] Generalization of Hermite functions by fractal interpolation, Journal of Approximation Theory 131 (2004), no. 1, 19-29.

[19] Smooth fractal interpolation, Journal of Inequalities and Applications, 2006 (2006), Article ID 78734, 20.

A. K. B. Chand: Mathematics Group, Birla Institute of Technology and Science, Pilani, Goa Campus, Vasco da Gama, Goa 403726, India

E-mail address: chand@bits-goa.ac.in

G. P. Kapoor: Department of Mathematics and Statistics, Indian Institute of Technology Kanpur, Kanpur 208 016, India

E-mail address: gp@iitk.ac.in 


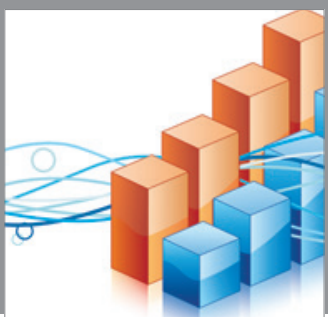

Advances in

Operations Research

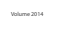

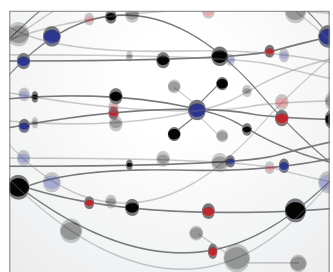

\section{The Scientific} World Journal
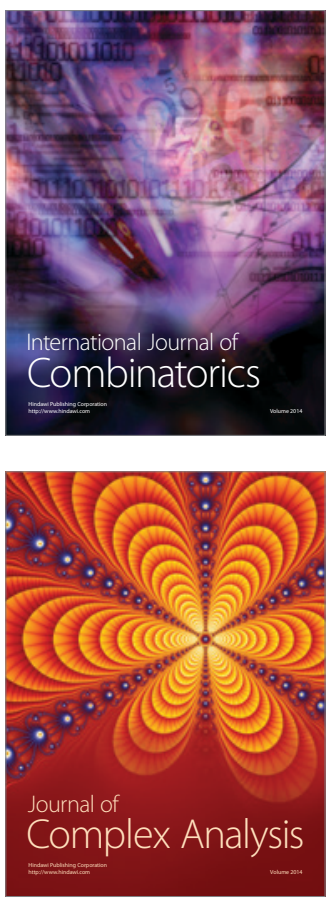

International Journal of

Mathematics and

Mathematical

Sciences
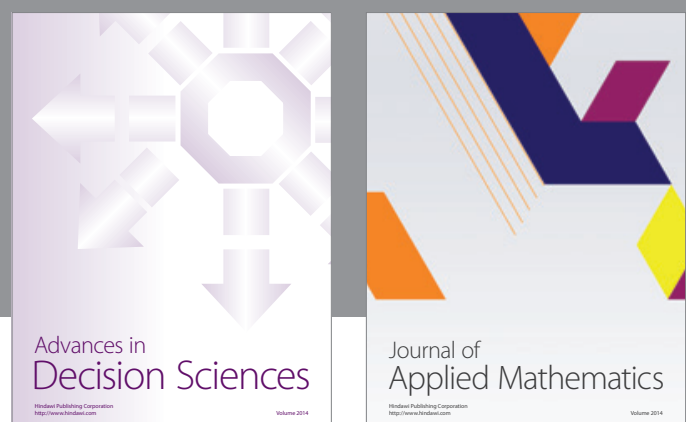

Journal of

Applied Mathematics
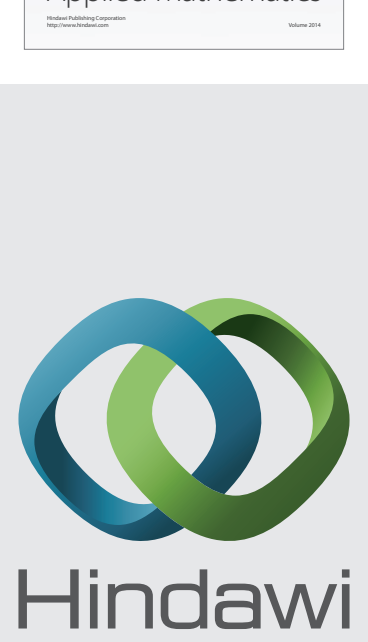

Submit your manuscripts at http://www.hindawi.com
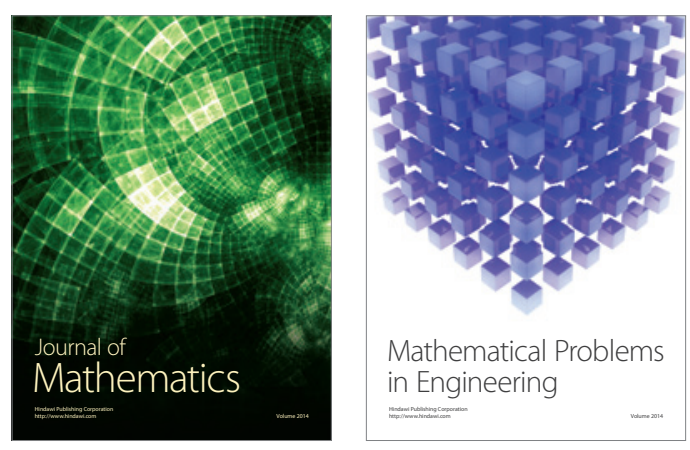

Mathematical Problems in Engineering
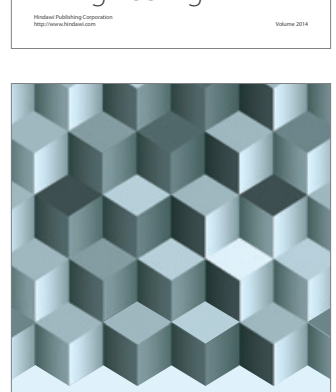

Journal of

Function Spaces
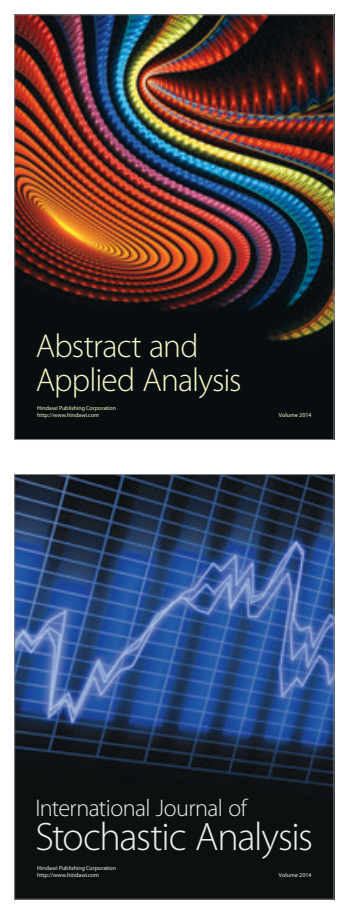

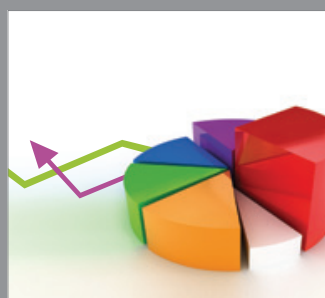

ournal of

Probability and Statistics

Promensencen
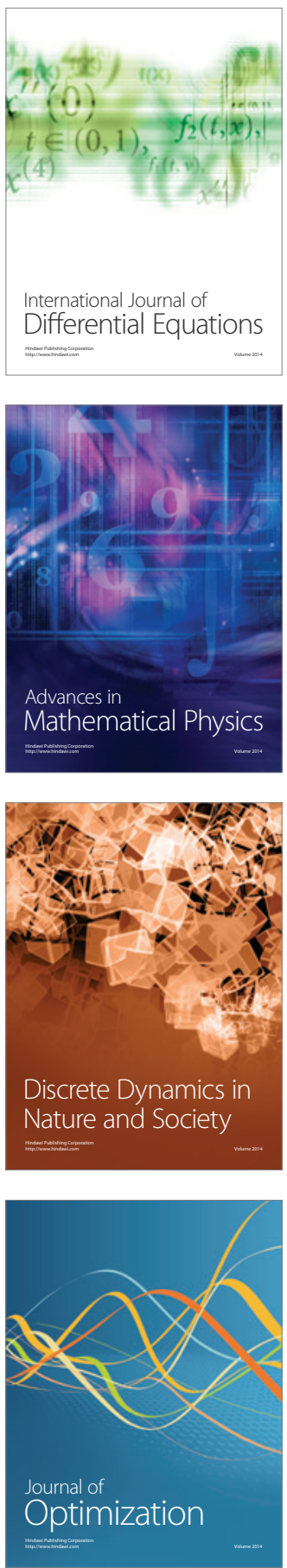